\title{
Well-graded families and the union-closed sets conjecture
}

\author{
Jeffrey Matayoshi \\ McGraw-Hill Education/ALEKS Corporation \\ Irvine, CA, U.S.A. \\ jeffrey.matayoshi@aleks.com
}

Submitted: Dec 12, 2018; Accepted: Feb 25, 2020; Published: Mar 20, 2020

(C) The author. Released under the CC BY license (International 4.0).

\begin{abstract}
The union-closed sets conjecture states that if a finite family of sets $\mathcal{F}$ is unionclosed, then there must be some element contained in at least half of the sets of $\mathcal{F}$. In this work we study the relationship between the union-closed sets conjecture and union-closed families that have the property of being well-graded. In doing so, we show how the density and other properties are affected by the extra structure contained in well-graded families, and we also give several conditions under which well-graded families satisfy the union-closed sets conjecture.
\end{abstract}

Mathematics Subject Classifications: 05D05, 06A07

\section{Introduction}

Let $\mathcal{F}$ be a finite family of finite sets with $|\mathcal{F}| \geqslant 2$. We say that $\mathcal{F}$ is union-closed if, for any $A, B \in \mathcal{F}$, we have $A \cup B \in \mathcal{F}$. Define $[n]:=\{1,2, \ldots, n\}$ and let $\mathcal{P}(n)=\mathcal{P}([n])$ be the power set of $[n]$. Supposing that $\mathcal{F}$ consists of subsets of $\mathcal{P}(n)$, define the degree of $x \in[n]$ as $d(x):=|\{K \in \mathcal{F} \mid x \in K\}|$. We say that $x$ is abundant in $\mathcal{F}$ if $d(x) \geqslant \frac{1}{2}|\mathcal{F}|$.

The union-closed sets conjecture, originally attributed to P. Frankl [20], states that if $\mathcal{F} \subseteq \mathcal{P}(n)$ is union-closed, then there must be some $x \in[n]$ that is contained in at least half of the sets of $\mathcal{F}$; in other words, there is at least one element in $[n]$ that is abundant in $\mathcal{F}$. Some of the more recent examples of work related to the conjecture are given by $[1,8,13,17,18]$, and for a thorough survey of the various results pertaining to the conjecture, as well as an introduction to many of the techniques used in these results, see [4]. In this work we will explore the connection between the union-closed sets conjecture and union-closed families that have the property of being well-graded. 
Definition 1. Let $\Delta$ denote the standard symmetric difference operation between sets. Given a family of sets, $\mathcal{F}$, a finite sequence of sets

$$
A=K_{0}, K_{1}, \ldots, K_{m}=B
$$

in $\mathcal{F}$ is called a (stepwise) path between $A$ and $B$ if $\left|K_{i-1} \Delta K_{i}\right|=1$ for all $i=1, \ldots, m$. If, additionally, $|A \Delta B|=m$, the sequence of sets is called a tight path between $A$ and $B$. The family $\mathcal{F}$ is well-graded if there exists a tight path between any $A, B \in \mathcal{F}$.

Well-graded families were first studied in [6] and have found widespread use in the area of knowledge space theory. Knowledge spaces are union-closed families containing the empty set that are used to model the knowledge of learners in various academic fields of study $[5,10,12]$. Such families that are also well-graded (known as learning spaces) have been effectively used in computerized tutoring systems. Thus, in addition to being of interest for their theoretical properties, well-graded families have become important for practical reasons; for example, they form the foundation of the artificial intelligence behind the ALEKS system [10,11].

In what follows we will study the properties of well-graded families and how they relate to the union-closed sets conjecture. In the case that a well-graded family contains the empty set, the conjecture follows easily. To see this, note that since both the empty set and the full set are contained in the family, there exists a tight path between them, and this tight path must contain a set with one element; thus, the result follows since it is well known that a family containing a singleton satisfies the conjecture [see, for example, 21]. On the other hand, if we no longer require that the family contains the empty set, the problem becomes more interesting since, in general, a well-graded union-closed family does not necessarily contain a singleton, or even a doubleton. However, intuitively, it would seem that well-graded families have more structure than families that are not well-graded, making it plausible that they are more likely to satisfy the union-closed sets conjecture. In this work we will prove several results that show, in some sense, this is true.

The outline of the paper is as follows. In Section 2 we begin by looking at how the well-graded property affects the density of a union-closed family. In Section 3 we use the concept of the outer fringe of a set to prove several results related to the union-closed sets conjecture; in particular, we show that if a minimal set $X$ (where 'minimal' is with respect to set inclusion) and its outer fringe are small enough, the family containing $X$ satisfies the conjecture. Finally, in Section 4 we prove a small extension of this last result by imposing an additional condition on the family containing the set $X$.

\section{Density of well-graded families}

For a family of sets $\mathcal{F}$, we say that $\cup_{K \in \mathcal{F}} K$ is the universe of $\mathcal{F}$. Also, let $\|\mathcal{F}\|:=$ $\sum_{K \in \mathcal{F}}|K|$ be the sum of the sizes of the sets in $\mathcal{F}$. In this section we will study the density of well-graded families with universe $[n]$, where we define density as in [22]. 
Definition 2. The density of a family $\mathcal{F}$ with universe $[n]$ is given by the formula

$$
\rho(\mathcal{F}):=\frac{\|\mathcal{F}\|}{|\mathcal{F}| \cdot n}=\frac{\sum_{x \in[n]} d(x)}{|\mathcal{F}| \cdot n}
$$

where $d(x)=|\{K \in \mathcal{F} \mid x \in K\}|$. In other words, it is the ratio of the average element degree to the size of $\mathcal{F}$. Note that $\rho(\mathcal{F}) \geqslant \frac{1}{2}$ implies that $\mathcal{F}$ contains an abundant element.

Letting $s_{n}$ be the minimum density of a union-closed family on the universe $[n]$, the values for $1 \leqslant n \leqslant 10$ were explicitly computed by Wójcik in [22]; the first six are given by $s_{1}=s_{2}=\frac{1}{2}, s_{3}=\frac{4}{9}, s_{4}=\frac{2}{5}, s_{5}=\frac{9}{25}$, and $s_{6}=\frac{1}{3}$. The next result will look at these values for well-graded families when $1 \leqslant n \leqslant 6$.

Proposition 3. Let $w_{n}$ be the minimum density of a well-graded union-closed family on the universe $[n]$. Then, we have $w_{1}=w_{2}=\frac{1}{2}, w_{3}=\frac{7}{15}, w_{4}=\frac{4}{9}, w_{5}=\frac{21}{50}$, and $w_{6}=\frac{9}{22}$.

Proof. Since the values of $w_{n}$, for $n=3, \ldots, 6$, are all computed in a similar fashion (but with each successive computation getting slightly more tedious), we will only show the steps for $w_{3}$. Following [22], we define

$$
a_{i}=|\{A \in \mathcal{F}:|A|=i\}|, \quad i=0,1, \ldots
$$

Note that $\rho(\{\varnothing,\{1\},\{2\},\{1,2\},\{1,2,3\}\})=\frac{7}{15}$, which implies that $w_{3} \leqslant \frac{7}{15}$. Suppose that $w_{3}<\frac{7}{15}$. Then, there exists a well-graded family $\mathcal{F}$ such that

$$
\frac{a_{1}+2 a_{2}+3}{3\left(a_{1}+a_{2}+2\right)}<\frac{7}{15}
$$

It then follows that $3 a_{2}+1<2 a_{1}$, which implies that $a_{2} \leqslant 1$. On the other hand, since $\mathcal{F}$ is well-graded, $a_{2} \geqslant 1$, leaving $a_{2}=1$ as the only possible value. However, since $3 a_{2}+1=3(1)+1<2 a_{1}$ we must have $a_{1}=3$; thus, since $\mathcal{F}$ is union-closed, this contradicts the assumption that $a_{2}=1$ (i.e., the union of each pair of sets in $a_{1}$ is in $\mathcal{F}$, which implies that $a_{2}=3$ ).

So, as we can see from the previous result, well-gradedness gets us slightly closer to the union-closed sets conjecture for $3 \leqslant n \leqslant 6$. However, we will see shortly that even in the case of a three-set, well-gradedness alone is not enough to guarantee that one of the three elements is abundant. Before presenting this result, we will need to introduce a few additional concepts.

Let $\mathcal{F}$ be a union-closed family of sets on $[n]$. For some $i \in[n]$, we say that an atom at $i$ is a set in $\mathcal{F}$ that contains $i$ and is minimal (with respect to set inclusion) among all such sets. The following result, originally from [14], gives us a way of checking for well-gradedness when $\mathcal{F}$ contains the empty set.

Theorem 4 (Koppen). Let $\mathcal{F}$ be a union-closed family of sets on $[n]$. Assume that $\mathcal{F}$ contains the empty set. Then, $\mathcal{F}$ is well-graded if and only if each atom in $\mathcal{F}$ is an atom at only one element of $[n]$. 
Another necessary concept, closely related to that of an atom, is the base of a unionclosed family.

Definition 5. The union-closure of a family of sets $\mathcal{G}$ is the family containing any set which is the union of any nonempty subfamily of $\mathcal{G}$. It follows that the empty set is in the union-closure of $\mathcal{G}$ if and only if it is in $\mathcal{G}$ itself. The base of a union-closed family $\mathcal{F}$ is a minimal subfamily $\mathcal{B}$ of $\mathcal{F}$ such that the union-closure of $\mathcal{B}$ is equal to $\mathcal{F}$.

It is known that, for a finite union-closed family, the base always exists, is unique, and is composed of all the atoms in $\mathcal{F}$ (see, for example, Section 3.4 in [10] for more details). We will also need the following definition and result from [9].

Definition 6. For any family of sets $\mathcal{G}$ and any set $X \in \mathcal{G}$, let $\mathcal{G} \backslash X$ denote the family of sets $\{Y \backslash X \mid Y \in \mathcal{G}\}$.

Theorem 7 (Eppstein, Falmagne, and Uzun). Let $\mathcal{B}$ be the base of a union-closed family $\mathcal{F}$. Then, $\mathcal{F}$ is well-graded if and only if, for each $X$ in $\mathcal{B}$, the union-closure of the family $\mathcal{B} \backslash X$ is a well-graded family.

Combining Theorems 4 and 7, we now have an efficient way of checking for the wellgradedness of any union-closed family, whether or not that family contains the empty set. Before giving our next result, we will need to introduce the following definition from [15].

Definition 8. A family of sets $\mathcal{F}$ is $X$-closed if for any nonempty subfamily $\mathcal{G}$ of $\mathcal{F}$, we have $\cap \mathcal{G} \in \mathcal{F}$ whenever $X \subseteq \cap \mathcal{G}$. When $\mathcal{F}$ is union-closed with base $\mathcal{B}$, we say that $\mathcal{F}$ is upper intersection-closed if $\mathcal{F}$ is $X$-closed for every $X \in \mathcal{B}$. In other words, any intersection of sets that includes an element of the base is contained in $\mathcal{F}$.

Following the notation introduced in [16] and later used in [4], given two families of sets $\mathcal{F}$ and $\mathcal{G}$ we define $\mathcal{F} \uplus \mathcal{G}:=\{K \cup L \mid K \in \mathcal{F}, L \in \mathcal{G}\}$. The next example shows that having a three-set in a well-graded family, even if that three-set is also $X$-closed, is not enough to guarantee that one of the three elements is abundant.

Example 9. Consider the following families of sets.

$$
\begin{aligned}
& \mathcal{A}_{1}=\{\varnothing,\{1\},\{2\}\} \\
& \mathcal{A}_{2}=\{\varnothing,\{1\},\{3\}\} \\
& \mathcal{A}_{3}=\{\varnothing,\{2\},\{3\}\} \\
& B_{i}=\{4,5,6, i+6\}, \quad i=1, \ldots, 6 \\
& \mathcal{B}=\{[6] \cup\{13\}, \\
& {[3],[4],[5],[6], } \\
& B_{1} \cup\{13\}, \ldots, B_{6} \cup\{13\}, \\
&\left.\mathcal{A}_{1} \uplus\left\{B_{1}, B_{4}\right\}, \mathcal{A}_{2} \uplus\left\{B_{2}, B_{5}\right\}, \mathcal{A}_{3} \uplus\left\{B_{3}, B_{6}\right\}\right\}
\end{aligned}
$$


We then have that $\mathcal{B}$ is the base of a union-closed family $\mathcal{F}$ on the universe [13], where $|\mathcal{B}|=29$. Using Theorem 4 , we can check that, for each $B \in \mathcal{B}$, the union closure of $\mathcal{B} \backslash B$ is well-graded; thus, by Theorem 7 it then follows that $\mathcal{F}$ is well-graded. Additionally, the family is $X$-closed for $X=[3]$. However, the family $\mathcal{F}$ contains 959 total sets, but each element of $X$ is contained in exactly 479 sets; thus, no element of the minimal three-set $X$ is abundant. ${ }^{1}$

Taking a slightly different approach, we will next look at the asymptotic behavior of $s_{n}$ and $w_{n}$ as $n$ grows. As mentioned in [22], for $r=\left\lceil\log _{2} n\right\rceil$ or $r=\left\lfloor\log _{2} n\right\rfloor$ the family $2^{[r]} \cup[n]$ has a density of $(1+o(1)) \frac{\log _{2} n}{2 n}$ as $n \rightarrow \infty$. Combining this with the following theorem from [2], we get that $s_{n}$ is on the order of $(1+o(1)) \frac{\log _{2} n}{2 n}$ as $n \rightarrow \infty$.

Theorem 10 (Balla). For all $n \in \mathbb{N}$,

$$
s_{n} \geqslant \frac{\log _{2} n}{2 n} .
$$

The next example, which is originally from [7], gives an upper bound on the asymptotic behavior of $w_{n}$ as $n \rightarrow \infty$.

Example 11 (Duffus and Sands). For $n \in \mathbb{N}$, let $r=\left\lceil\log _{2}\left(\frac{n^{2}}{\log _{2} n^{2}}\right)\right\rceil$ or $r=\left\lfloor\log _{2}\left(\frac{n^{2}}{\log _{2} n^{2}}\right)\right\rfloor$. Then, the family given by

$$
\mathcal{F}=\mathcal{P}(r) \cup\{[i] \mid i=r+1, \ldots, n\}
$$

is union-closed and well-graded, with

$$
|\mathcal{F}|=2^{r}+n-r
$$

and

$$
\|\mathcal{F}\|=r 2^{r-1}+\frac{n(n+1)}{2}-\frac{r(r+1)}{2} .
$$

Thus, we have

$$
\begin{aligned}
\rho(\mathcal{F}) & =\frac{r 2^{r-1}+\frac{n(n+1)}{2}-\frac{r(r+1)}{2}}{n\left(2^{r}+n-r\right)} \\
& =(1+o(1)) \frac{\log _{2} n}{n} \text { as } n \rightarrow \infty .
\end{aligned}
$$

The rest of this section will be devoted to showing that $w_{n}$ is also bounded below by the same asymptotic value as $n \rightarrow \infty$; this would then imply that, as $n \rightarrow \infty$, the limiting value of $w_{n}$ is larger than the limiting value of $s_{n}$ by a factor of 2 . To derive this lower bound, we will need to introduce a few additional concepts. Let $\mathbb{N}^{(<\infty)}$ be the collection

\footnotetext{
${ }^{1}$ A Python module that generates the example family and performs these computations is available at https://github.com/jmatayoshi/uc-conjecture-well-graded.
} 
of all finite sets of positive integers. For $A, B \in \mathbb{N}^{(<\infty)}$, we define the colex order on $\mathbb{N}^{(<\infty)}$ as the linear order $<$ given by

$$
A<B \Longleftrightarrow \max (A \Delta B) \in B
$$

The beginning of the colex order has the form

$$
\begin{aligned}
\varnothing<1<2<12<3<13<23<123 & <4 \\
& <14<24<124<34<134<234<1234,
\end{aligned}
$$

where for convenience we have written, for example, 123 for the set $\{1,2,3\}$. Following [3], we will write $\mathcal{I}(m)$ for the initial segment of the colex order of length $m$. We will say that a family $\mathcal{F}$ is separating if for any $i$ and $j$ in $[n]$, there is a set in $\mathcal{F}$ that contains exactly one of $i$ or $j$. Additionally, a separating family $\mathcal{F}$ is normal if each $i$ in $[n]$ is contained in at least one set of $\mathcal{F}$. The following result from [3] identifies the normal families of minimum average set size.

Theorem 12 (Balla, Bollobas and Eccles). For $n, k \in \mathbb{N}$ such that $k \leqslant 2^{n}$, define the set $\mathcal{F}(k):=\mathcal{P}(r-1) \cup\{A+r \mid A \in \mathcal{U}\}$, where $\mathcal{U}=\mathcal{P}(r-1) \backslash \mathcal{I}\left(2^{r}-k\right)$ and $r=\left\lceil\log _{2}(k)\right\rceil$. Then, for $m \in \mathbb{N}$ let $f(n, m)$ be defined by

$$
f(n, m)=\min (\|\mathcal{F}\|),
$$

where the minimum is taken over all normal union-closed families in $\mathcal{P}(n)$ which consist of $m$ sets. Let $\mathcal{F}(n, k)$ be given by

$$
\mathcal{F}(n, k)=\mathcal{F}(k) \cup\{[i]: 1 \leqslant i \leqslant n\} .
$$

Then for any integers $m$ and $n$ with $n+1 \leqslant m \leqslant 2^{n}$ there exists $k$ such that $|\mathcal{F}(n, k)|=m$, and we then have

$$
f(n, m)=\|\mathcal{F}(n, k)\| .
$$

The following lemma, also from [3], will be useful.

Lemma 13 (Balla, Bollobas and Eccles). For all $m \in \mathbb{N}$,

$$
\frac{m\left(\log _{2}(m)-1\right)}{2}<\|\mathcal{I}(m)\| \leqslant \frac{m\left(\log _{2}(m)\right)}{2} .
$$

For a well-graded union-closed family with universe $[n]$, the next result gives a lower bound for the density when the number of sets in the family is small.

Lemma 14. Let $\mathcal{F}$ be a well-graded union-closed family with universe [n], and assume that $|\mathcal{F}| \leqslant n$. Then,

$$
\rho(\mathcal{F}) \geqslant \frac{1}{2}
$$


Proof. Let $m=|\mathcal{F}| \leqslant n$. By assumption, $\mathcal{F}$ contains $[n]$ and is a well-graded union-closed family of size $m$. Suppose that $\mathcal{M}$ is a family of minimal density over all such families $\mathcal{F}$. Let $M \in \mathcal{M}$ be a set of minimal size (i.e., for any other $K \in \mathcal{M},|M| \leqslant|K|$ ). Since $\mathcal{M}$ is well-graded, there must be a tight path from $M$ to $[n]$ of the form $M=$ $M_{0}, M_{1}, \ldots, M_{j}=[n]$, where $j<m$ and $\left|M_{i}\right|+1=\left|M_{i+1}\right|, i=0, \ldots, j-1$. Now, we claim that $|M|=n-m+1$. To show this, we begin by observing that we cannot have $|M|<n-m+1$; otherwise, the tight path from $M$ to $[n]$ would contain more sets than the entire family $\mathcal{M}$.

Next, suppose that $|M|>n-m+1$. In this case, the tight path from $M$ to $[n]$ consists of $n-|M|+1$ sets, with the remaining $m-(n-|M|+1)$ sets in $\mathcal{M}$ each containing $|M|$ or more elements (since $M$ is a set of minimal size). In comparison, consider a family, $\widetilde{\mathcal{M}}$, consisting solely of a tight path that begins at a minimal set of size $n-m+1$ and that ends at $[n]$. Putting the sets in $\widetilde{\mathcal{M}}$ in increasing order based on their sizes, the last $n-|M|+1$ sets in $\widetilde{\mathcal{M}}$ have a total size equal to the tight path from $M$ to $[n]$. However, the remaining $m-(n-|M|+1)$ sets in $\widetilde{\mathcal{M}}$ all have fewer than $|M|$ elements, contradicting the assumption that $\mathcal{M}$ is a family of minimal density (and, hence, the assumption that $|M|>n-m+1)$.

Thus, we have now shown that the family of minimal density, $\mathcal{M}$, consists solely of a tight path from a set $M$ to $[n]$, where $|M|=n-m+1$. It follows that

$$
\begin{aligned}
\rho(\mathcal{F}) & \geqslant \frac{\| \mathcal{M}||}{n|\mathcal{M}|} \\
& =\frac{\sum_{i=0}^{m-1}\left|M_{i}\right|}{n \cdot m} \\
& =\frac{\frac{n(n+1)}{2}-\frac{(n-m)(n-m+1)}{2}}{n \cdot m} \\
& \geqslant \frac{1}{2} .
\end{aligned}
$$

Our next result will show that, to find a well-graded family of minimum density, it suffices to consider only normal well-graded families.

Lemma 15. Let $\mathcal{F}$ be a well-graded union-closed family with universe $[n]$, and suppose that $|\mathcal{F}| \geqslant n+1$. Then, there exists a well-graded union-closed family $\widetilde{\mathcal{F}}$ on $[n]$ such that $\widetilde{\mathcal{F}}$ is normal and

$$
\rho(\widetilde{\mathcal{F}}) \leqslant \rho(\mathcal{F}) .
$$

Proof. Suppose that $\mathcal{F}$ is not separating (otherwise, we are done). Then, there exist $i, j \in[n]$ such that for any $K \in \mathcal{F}, i \in K$ if and only if $j \in K$. Now, we claim that $i$ and $j$ must be contained in every set of $\mathcal{F}$. Suppose they are not. Then, there exists $K \in \mathcal{F}$ such that $K \cap\{i, j\}=\varnothing$. Note, however, that this contradicts the assumption that $\mathcal{F}$ is well-graded since it is not possible for a tight path to exist between $K$ and any set containing $i$ and $j$; that is, since $i$ and $j$ can never appear separately, it is not possible to 
go in a single step from a set without $i$ and $j$ to a set with both $i$ and $j$. Thus, $\{i, j\} \subseteq K$ for any $K \in \mathcal{F}$.

Now that we have shown $i$ and $j$ are contained in every set of $\mathcal{F}$, consider the unionclosed family

$$
\mathcal{F}_{j}=\{K \backslash\{j\} \mid K \in \mathcal{F}\} \cup\{[n]\} .
$$

The family $\mathcal{F}_{j}$ is well-graded, contains sets with $i$ and not $j$, and contains $[n]$ as a set. Furthermore, since $|\mathcal{F}| \geqslant n+1$, we have

$$
\left\|\mathcal{F}_{j}\right\|=\|\mathcal{F}\|-|\mathcal{F}|+n \leqslant\|\mathcal{F}\|-1<\|\mathcal{F}\|,
$$

which, combined with the fact that $\left|\mathcal{F}_{j}\right|=|\mathcal{F}|+1$, implies that $\rho\left(\mathcal{F}_{j}\right)<\rho(\mathcal{F})$.

As a final step, note that if $\mathcal{F}_{j}$ is not separating, we can iteratively repeat this same procedure until we eventually arrive at a normal well-graded family, as claimed.

Taken together, Theorem 12 and Lemma 15 tell us that for $m \geqslant n+1$, the well-graded family of minimum average size is given by $\mathcal{F}(n, k)$, for some $k \leqslant n$; thus, as our next result will show, we can bound $w_{n}$ from below by finding a lower bound for the density of $\mathcal{F}(n, k)$.

Theorem 16. Let $w_{n}$ be the minimum density of a well-graded union-closed family on the universe $[n]$. Then, $w_{n}$ is on the order of $(1+o(1)) \frac{\log _{2} n}{n}$ as $n \rightarrow \infty$.

Proof. By Lemma 14, it suffices to consider $\mathcal{F}$ such that $|\mathcal{F}| \geqslant n+1$. Thus, from Theorem 12 , for integers $n$ and $m$ with $n+1 \leqslant m \leqslant 2^{n}$ we know that the well-graded family of minimum density is given by $\mathcal{F}(n, k)$, for some $k$ in $\left[n+1,2^{n}\right]$. Combined with Example 11 , the result will follow if we can show that $\mathcal{F}(n, k) \geqslant(1+o(1)) \frac{\log _{2} n}{n}$ as $n \rightarrow \infty$, for any $k$ in $\left[n+1,2^{n}\right]$.

Let $r=\left\lceil\log _{2}\left(\frac{n^{2}}{\log _{2} n^{2}}\right)\right\rceil$ and $k=2^{r}-\widetilde{k}$, where $0 \leqslant \widetilde{k}<2^{r-1}$. Letting $\mathcal{U}=\{\mathcal{P}(r-1) \backslash$ $\left.\left.\mathcal{I}\left(2^{r}-k\right)\right)\right\}$, it follows that

$$
\mathcal{P}(r-1) \cup\{A+r: A \in \mathcal{U}\}=\mathcal{F}(k) .
$$

We then have

$$
\begin{aligned}
|\mathcal{F}(n, k)| & =|\mathcal{P}(r-1)|+|\{A+r: A \in \mathcal{U}\}|+(n-r) \\
& =2^{r-1}+|\mathcal{U}|+(n-r) \\
& =2^{r}-\widetilde{k}+(n-r),
\end{aligned}
$$

and

$$
\begin{aligned}
\|\mathcal{F}(n, k)\| & =\|\mathcal{P}(r-1)\|+\|\{A+r: A \in \mathcal{U}\}\|+\sum_{i=r+1}^{n} i \\
& =\frac{1}{2}(r-1) 2^{r-1}+\|\{A+r: A \in \mathcal{U}\}\|+\frac{1}{2}(n-r)(n+r+1),
\end{aligned}
$$


where in (2.8) we used the fact that $|\mathcal{U}|=2^{r-1}-\widetilde{k}$. Next, note that

$$
\begin{aligned}
\|\{A+r: A \in \mathcal{U}\}\| & =\|\mathcal{P}(r-1)\|-\left\|\mathcal{I}\left(2^{r}-k\right)\right\|+|\mathcal{U}| \\
& =\frac{1}{2}(r-1) 2^{r-1}-\|\mathcal{I}(\widetilde{k})\|+|\mathcal{U}|
\end{aligned}
$$

Combining (2.9) and (2.10) with Lemma 13, we have

$$
\begin{aligned}
\|\mathcal{F}(n, k)\| & =(r-1) 2^{r-1}-\|\mathcal{I}(\widetilde{k})\|+|\mathcal{U}|+\frac{1}{2}(n-r)(n+r+1) \\
& \geqslant(r-1) 2^{r-1}-\frac{1}{2} \widetilde{k} \log _{2}(\widetilde{k})+|\mathcal{U}|+\frac{1}{2}(n-r)(n+r+1) \\
& =(r) 2^{r-1}-\frac{1}{2} \widetilde{k} \log _{2}(\widetilde{k})-\widetilde{k}+\frac{1}{2}(n-r)(n+r+1),
\end{aligned}
$$

where the last equality again uses the fact that $|\mathcal{U}|=2^{r-1}-\widetilde{k}$. Using (2.8) and (2.11), we can bound the density of $\mathcal{F}(n, k)$ from below with the inequality

$$
\rho(\mathcal{F}(n, k)) \geqslant \frac{(r) 2^{r-1}-\frac{1}{2} \widetilde{k} \log _{2}(\widetilde{k})-\widetilde{k}+\frac{1}{2}(n-r)(n+r+1)}{n\left(2^{r}-\widetilde{k}+(n-r)\right)} .
$$

Notice that, when viewed as a function of $\widetilde{k},(2.12)$ has no critical points on the interval $\left(0,2^{r-1}\right)$, which implies that the minimum must occur at either 0 or $2^{r-1}$. Thus, we now have

$$
\rho(\mathcal{F}(n, k)) \geqslant \frac{(r) 2^{r-1}+\frac{1}{2}(n-r)(n+r+1)}{n\left(2^{r}+(n-r)\right)},
$$

where $k=2^{r}$ for some $r \in[2,3, \ldots, n]$; note that the right-hand side of $(2.13)$ is equal to the density of the family from Example 11. Thus, letting $g(r)$ be equal to the righthand side of (2.13), the discussion in Example 11 shows that, for $r=\left\lceil\log _{2}\left(\frac{n^{2}}{\log _{2} n^{2}}\right)\right\rceil$ or $r=\left\lfloor\log _{2}\left(\frac{n^{2}}{\log _{2} n^{2}}\right)\right\rfloor, g(r)=(1+o(1)) \frac{\log _{2} n}{n}$ as $n \rightarrow \infty$.

It remains to show that $g(r)$ is bounded below by $(1+o(1)) \frac{\log _{2} n}{n}$ as $n \rightarrow \infty$, for all $r$. To that end, note that for any $r>\left\lceil\log _{2}\left(\frac{n^{2}}{\log _{2} n^{2}}\right)\right\rceil$, the first term in the numerator of $g(r)$ dominates with a value bounded below by $(1+o(1)) \frac{\log _{2} n}{n}$ as $n \rightarrow \infty$. On the other hand, for $r<\left\lfloor\log _{2}\left(\frac{n^{2}}{\log _{2} n^{2}}\right)\right\rfloor$, the second term in the numerator dominates, again with a value bounded below by $(1+o(1)) \frac{\log _{2} n}{n}$ as $n \rightarrow \infty$.

\section{The outer fringe}

Given a set $X$ in a union-closed family $\mathcal{F}$, consider an element $i \notin X$ such that $X \cup\{i\} \in \mathcal{F}$. If $\mathcal{F}$ is well-graded, and if $X \subset[n]$, such an $i$ must exist; this follows from the fact that there exists a tight path in $\mathcal{F}$ from $X$ to $[n]$. In the knowledge spaces literature, an element 
with such a property is said to be in the outer fringe of the set $X$, denoted by $X^{\mathcal{O}}$, and for convenience we will use that terminology here. In this section, given a minimal set $X$ (where 'minimal' is with respect to set inclusion) in a well-graded family of sets, we will prove several results pertaining to the elements in the outer fringe of $X$. To do this, we will need to make use of the following lemma.

Lemma 17. Let $\mathcal{F}$ be a well-graded union-closed family of sets, and let $X$ be a set in $\mathcal{F}$. Then, for any $K \in \mathcal{F}$ such that $K \backslash X \neq \varnothing$, we have $K \cap X^{\mathcal{O}} \neq \varnothing$.

Proof. Since $\mathcal{F}$ is well-graded, there exists a tight path from $X$ to $K \cup X$. Let $L$ be the first set of this tight path that is not equal to $X$; thus, $L$ has the form $X \cup\{i\}$, for some $i \in[n]$, which implies that $i \in X^{\mathcal{O}}$. Also, $i \in K \cup X$ since $L \subset K \cup X$; thus, combined with the fact that $X \cap X^{\mathcal{O}}=\varnothing$, it must then be the case that $i \in K$.

Theorem 18. Let $X$ be a minimal set in a well-graded union-closed family $\mathcal{F}$, where $X \subset[n]$, and suppose that $\left|X^{\mathcal{O}}\right| \leqslant 2$. Then, one of the elements in $X^{\mathcal{O}}$ is abundant.

Proof. First, suppose that $i$ is the only element in $X^{\mathcal{O}}$ and let $K$ be a set in $\mathcal{F}$. Since $X$ is minimal in $\mathcal{F}$, either $K=X$, or $K \backslash X \neq \varnothing$. Assume the latter. Then, by Lemma 17, $K$ must contain an element of $X^{\mathcal{O}}$, and since we are assuming that $i$ is the only element in $X^{\mathcal{O}}$, we must have $i \in K$. So, we have now shown that the only set in $\mathcal{F}$ that does not contain $i$ is $X$, from which it follows that $i$ is abundant.

Next, suppose that $X^{\mathcal{O}}=\{i, j\}$. Consider the following partition of $\mathcal{F}$ :

$$
\begin{aligned}
& A_{i, j}=\{K \in \mathcal{F} \mid i, j \in K\} \\
& A_{i, \bar{j}}=\{K \in \mathcal{F} \mid i \in K, j \notin K\} \\
& A_{\bar{i}, j}=\{K \in \mathcal{F} \mid i \notin K, j \in K\} \\
& A_{\bar{i}, \bar{j}}=\{K \in \mathcal{F} \mid i, j \notin K\} .
\end{aligned}
$$

Without loss of generality, assume that $\left|A_{i, \bar{j}}\right| \geqslant\left|A_{\bar{i}, j}\right|$. By the argument in the previous paragraph, we know that $A_{\bar{i}, \bar{j}}=\{X\}$. Also, since $[n] \in A_{i, j}$ we have $\left|A_{i, j}\right| \geqslant 1=\left|A_{\bar{i}, \bar{j}}\right|$. Thus, it follows that

$$
\begin{aligned}
d(i) & =\frac{\left|A_{i, j}\right|+\left|A_{i, \bar{j}}\right|}{\left|A_{i, j}\right|+\left|A_{i, \bar{j}}\right|+\left|A_{\bar{i}, j}\right|+\left|A_{\bar{i}, \bar{j}}\right|} \\
& =\frac{\left|A_{i, j}\right|+\left|A_{i, \bar{j}}\right|}{\left|A_{i, j}\right|+\left|A_{i, \bar{j}}\right|+\left|A_{\bar{i}, j}\right|+1} \\
& \geqslant \frac{\left|A_{i, j}\right|+\left|A_{i, \bar{j}}\right|}{\left|A_{i, j}\right|+2\left|A_{i, \bar{j}}\right|+1} \\
& \geqslant \frac{1}{2},
\end{aligned}
$$

as claimed. 
Corollary 19. Let $X$ be a minimal set in a well-graded union-closed family $\mathcal{F}$, where $X \subset[n]$, and suppose that $\left|X \cup X^{\mathcal{O}}\right| \leqslant 5$. Then, one of the elements in $X \cup X^{\mathcal{O}}$ is abundant.

Proof. If $|X| \leqslant 2, X$ must contain an abundant element. If $|X| \geqslant 3$, then $\left|X^{\mathcal{O}}\right| \leqslant 2$ since $\left|X \cup X^{\mathcal{O}}\right| \leqslant 5$, and from Theorem 18 it follows that $X^{\mathcal{O}}$ must contain an abundant element.

The following example shows that having three elements in the outer fringe of a minimal set in a well-graded family is not enough to guarantee that one of the elements is abundant.

Example 20. Consider the following family of sets on the universe [6]:

$$
\begin{aligned}
\mathcal{F}=\{\{1,2,3\},\{1,2,4\}, & \{1,2,3,4\},\{2,3,5\},\{1,2,3,5\},\{1,3,6\},\{1,2,3,6\}, \\
& \{1,2,3,4,5\},\{1,2,3,4,6\},\{1,2,3,5,6\},\{1,2,3,4,5,6\}\} .
\end{aligned}
$$

Note that $\mathcal{F}$ is union-closed and well-graded, and note also that $X=\{1,2,3\}$ is minimal in $\mathcal{F}$ with an outer fringe consisting of $X^{\mathcal{O}}=\{4,5,6\}$. However, since each element of $X^{\mathcal{O}}$ appears in only 5 of the 11 sets in $\mathcal{F}, X^{\mathcal{O}}$ does not contain an abundant element.

For a set $X$ in a well-graded union-closed family $\mathcal{F}$, we have now seen examples where one of $X$ or $X^{\mathcal{O}}$ contains an abundant element, but not the other; however, we have yet to see an example where neither of the two contains an abundant element. The next result gives a set of conditions under which we can always expect an abundant element in $X \cup X^{\mathcal{O}}$.

Theorem 21. Let $X$ be a set in a well-graded union-closed family $\mathcal{F}$. Suppose also that $\mathcal{F}$ is $X$-closed. If $\mathcal{F}$ contains an abundant element, then one of the elements in $X \cup X^{\mathcal{O}}$ must be abundant.

Proof. Let $i$ be abundant in $\mathcal{F}$, and assume that $i \notin X \cup X^{\mathcal{O}}$ (otherwise, we are done). Let $\mathcal{A}_{i}=\{A \in \mathcal{F} \mid i \in A\}$. Then, since $\mathcal{F}$ is $X$-closed, we have that

$$
K=\bigcap_{A \in \mathcal{A}_{i}}(A \cup X)
$$

is a set in $\mathcal{F}$ containing $X$. We first claim that $K \cap X^{\mathcal{O}}$ is non-empty. To see this, note that there must be a tight path in $\mathcal{F}$ from $X$ to $K$. Thus, since $X \subset K$, the first set on this path has the form $X \cup\{j\}$, for some $j \in[n]$, which in turn implies that $j \in X^{\mathcal{O}}$; furthermore, $j \neq i$ since we are assuming that $i \notin X^{\mathcal{O}}$.

Next, we claim that for any $L \in \mathcal{F}, j \in L$ whenever $i \in L$. Assume the opposite; that is, suppose there exists $L \in \mathcal{F}$ such that $i \in L$ but $j \notin L$. Since $L \cup X \in \mathcal{A}_{i}$, this would imply that $j \notin K$, contradicting our assumption that $X \cup\{j\}$ is part of a tight path from $X$ to $K$. Thus, $j$ appears in at least as many sets as $i$, from which it follows that $j$ is abundant as well. 
Thus, if the union-closed sets conjecture holds, Theorem 21 tells us that one of the elements in $X \cup X^{\mathcal{O}}$ will be abundant whenever $\mathcal{F}$ is both well-graded and $X$-closed. Generalizing these results further, we make the following conjecture for all well-graded families.

Conjecture 22. Let $\mathcal{F}$ be a well-graded union-closed family, and let $X$ be a set in $\mathcal{F}$. Then, one of the elements in $X \cup X^{\mathcal{O}}$ is abundant.

\section{Density of a set and its outer fringe}

Given a set $X$ in a well-graded family $\mathcal{F}$, in this section we will analyze the behavior of the density of $X \cup X^{\mathcal{O}}$ under various conditions. Based on these results, our final theorem will show that given a minimal set $X$ in an $X$-closed family $\mathcal{F}$, the union-closed sets conjecture is satisfied if $\left|X \cup X^{\mathcal{O}}\right| \leqslant 6$, extending Corollary 19. To start, we will need the following result from [19], which gives a lower bound on the average set size of a union-closed family.

Theorem 23 (Reimer). Let $\mathcal{F}$ be a union-closed family with universe $[n]$. We then have

$$
\frac{\sum_{K \in \mathcal{F}}|K|}{|\mathcal{F}|} \geqslant \frac{1}{2} \log _{2}(|\mathcal{F}|) .
$$

For a given family $\mathcal{F}$ and a subset of elements $Y$, we will be interested in computing the density of $\mathcal{F}$ restricted to the elements in $Y$; that is, we are interested in the value of

$$
\rho_{Y}(\mathcal{F})=\frac{\|\mathcal{F}\|_{Y}}{|\mathcal{F}| \cdot|Y|}
$$

where $\|\mathcal{F}\|_{Y}=\sum_{K \in \mathcal{F}}|K \cap Y|$. Note that if $\rho_{Y}(\mathcal{F}) \geqslant \frac{1}{2}$, then one of the elements in $Y$ must be abundant in $\mathcal{F}$.

The following lemma, which is an application of Theorem 23, will be useful.

Lemma 24. Let $\mathcal{G}$ be a union-closed family with universe $[n]$. Let $L \subseteq[n]$, and suppose that for any $K \in \mathcal{G}$ we have $L \subseteq K$. We then have

$$
\frac{\sum_{K \in \mathcal{G}}|K|}{|\mathcal{G}|} \geqslant \frac{1}{2} \log _{2}(|\mathcal{G}|)+|L|
$$

Proof. Define the family $\mathcal{G}_{L}=\{K \backslash L \mid K \in \mathcal{G}\}$. Note that since $\mathcal{G}_{L}$ is a union-closed family, we can apply Theorem 23 to get the following bound:

$$
\sum_{K \in \mathcal{G}_{L}}|K| \geqslant \frac{\left|\mathcal{G}_{L}\right|}{2} \log _{2}\left(\left|\mathcal{G}_{L}\right|\right) .
$$


Next, observe that the mapping $f: \mathcal{G} \rightarrow \mathcal{G}_{L}$ defined by $f(K)=K \backslash L$ defines a bijection from $\mathcal{G}$ to $\mathcal{G}_{L}$. Combining this with (4.4), we get

$$
\begin{aligned}
\sum_{K \in \mathcal{G}}|K| & =\sum_{K \in \mathcal{G}_{L}}|K|+|\mathcal{G}| \cdot|L| \\
& \geqslant \frac{\left|\mathcal{G}_{L}\right|}{2} \log _{2}\left(\left|\mathcal{G}_{L}\right|\right)+|\mathcal{G}| \cdot|L| \\
& =\frac{|\mathcal{G}|}{2} \log _{2}(|\mathcal{G}|)+|\mathcal{G}| \cdot|L|,
\end{aligned}
$$

as claimed.

Lemma 25. Let $X$ be a set in a well-graded union-closed family $\mathcal{F}$, and let $\widetilde{X}=X \cup X^{\mathcal{O}}$. For sets $K, L \in \mathcal{F}$, define the equivalence relation $R$ as

$$
(K, L) \in R \Longleftrightarrow K \cup \widetilde{X}=L \cup \widetilde{X} .
$$

We then have the equivalence class

$$
[K]_{R}=\{L \in \mathcal{F} \mid(K, L) \in R\},
$$

where $[K]_{R}$ is a well-graded union-closed family. Furthermore, if $\mathcal{F}$ is also $X$-closed, and if $K \backslash \widetilde{X} \neq \varnothing$, then there exists $q \in X^{\mathcal{O}}$ such that $q \in M$ for any $M \in[K]_{R}$.

Proof. It is clear that $[K]_{R}$ is union-closed. To check that it is well-graded, for $L, M \in$ $[K]_{R}$ we must show that there exists a tight path from $L$ to $M$. Since $\mathcal{F}$ is well-graded, there exists a tight path $L=L_{0}, L_{1}, \ldots, L_{m}=M$ in $\mathcal{F}$ from $L$ to $M$. To show that this path exists in $[K]_{R}$, we simply need to show that $L_{i} \cup \widetilde{X}=K \cup \widetilde{X}$, for $i=1, \ldots m-1$. To that end, we first observe that since $L$ and $M$ are both in $[K]_{R}$, by (4.5) they can only differ on elements in $\widetilde{X}$. Thus, it follows that each $L_{i}, i=1, \ldots, m-1$ must also differ from $L$ and $M$ only on elements in $\widetilde{X}$, and the result then follows.

Next, assume also that $\mathcal{F}$ is $X$-closed and that $K \backslash \widetilde{X} \neq \varnothing$. To show that there exists some $q \in X^{\mathcal{O}}$ that is in every set of $[K]_{R}$, we will proceed by contradiction. That is, suppose that for each $q \in X^{\mathcal{O}}$, there exists some $K_{\bar{q}} \in[K]_{R}$ such that $q \notin K_{\bar{q}}$. Furthermore, since $K_{\bar{q}} \cup X \in[K]_{R}$ as well, without loss of generality we can assume that $X \subset K_{\bar{q}}$. Thus, we can define

$$
I=\bigcap_{q \in X^{\mathcal{O}}} K_{\bar{q}}
$$

where $I \cap X^{\mathcal{O}}=\varnothing$, and $I \in \mathcal{F}$ since $\mathcal{F}$ is $X$-closed. Since $K \backslash \widetilde{X} \neq \varnothing$, it follows that $I \backslash \widetilde{X} \neq \varnothing$ as well; that is, because the sets in $[K]_{R}$ only differ on elements in $\tilde{X}$, the elements in $K \backslash \widetilde{X}$ must be in every set of $[K]_{R}$. Now, note that by the well-gradedness of $\mathcal{F}$, there exists a tight path $X=X_{0}, X_{1}, \ldots, X_{m}=I$ from $X$ to $I$. Furthermore, since $X$ is strictly contained in $I$ (and, hence, in $X_{1}$ ), it follows that $X_{1}=X \cup\{j\}$, for some $j \in[n]$; thus, $j \in X^{\mathcal{O}}$, contradicting the assumption that $I \cap X^{\mathcal{O}}=\varnothing$. 
Lemma 26. Let $X$ be a set in a well-graded union-closed family $\mathcal{F}$, and assume also that $\mathcal{F}$ is $X$-closed and that $|X|=3$. Let $\widetilde{X}=X \cup X^{\mathcal{O}}$. Then, for any $K \in \mathcal{F}$ such that $K \backslash \widetilde{X} \neq \varnothing$, we have $\rho_{\widetilde{X}}\left([K]_{R}\right) \geqslant \frac{1}{2}$.

Proof. Without loss of generality, assume $X=\{1,2,3\}$. To start, observe that we can partition $[K]_{R}$ into the following union-closed families:

$$
\begin{aligned}
& K_{1}=\left\{L \in[K]_{R} \mid X \subseteq L\right\} \\
& K_{2}=\left\{L \in[K]_{R} \mid 1 \notin L\right\} \\
& K_{3}=\left\{L \in[K]_{R} \mid 2 \notin L, 1 \in L\right\} \\
& K_{4}=\left\{L \in[K]_{R} \mid 3 \notin L,\{1,2\} \subset L\right\} .
\end{aligned}
$$

By assumption, $K \backslash \tilde{X} \neq \varnothing$. Let $M \subseteq K \cup \widetilde{X}$ be the largest set of elements in $K \cup \tilde{X}$ such that $M \subseteq L$ for any $L \in[K]_{R}$; note that since $\mathcal{F}$ is $X$-closed, by Lemma 25 we have $\left|M \cap X^{\mathcal{O}}\right|=k \geqslant 1$. We claim that $K_{1}$ is equal to $(M \cup X) \uplus \mathcal{P}\left(X^{\mathcal{O}} \backslash M\right)$. Since $[K]_{R}$ is union-closed, the claim will follow if we can show that, for any $r \in X^{\mathcal{O}} \backslash M$, the set $M \cup X \cup\{r\}$ is contained in $[K]_{R}$.

To that end, note that the sets in $[K]_{R}$ differ only on the elements in $(K \cup \tilde{X}) \backslash M \subset \widetilde{X}$. Next, define the set

$$
I_{M}=\bigcap_{q \in X^{\mathcal{O}} \backslash M} K_{\bar{q}}
$$

where each $K_{\bar{q}}$ is defined as in the proof of Lemma 25 (and where we are again assuming that each $K_{\bar{q}}$ contains $X$ ). We claim that $I_{M}=M \cup X$. To see this, we first note that the relation $I_{M} \supseteq M \cup X$ is clear, as each $K_{\bar{q}}$ contains $X$, while $M$ is contained in every set of $[K]_{R}$. Next, let $r \in I_{M}$. Then, by construction $r \notin X^{\mathcal{O}} \backslash M$; thus, since the universe of $[K]_{R}$ is given by $M \cup X \cup X^{\mathcal{O}}$, either $r \in X$ or $r \in M$, and it follows that $I_{M} \subseteq M \cup X$. Combining these two arguments, we have now shown that $I_{M}=M \cup X$.

Next, since $\mathcal{F}$ is $X$-closed, we have $M \cup X=I_{M} \in \mathcal{F}$. Finally, we note that $X \cup\{r\} \in$ $\mathcal{F}$, since $r \in X^{\mathcal{O}}$, and the fact that $\mathcal{F}$ is union-closed implies that $M \cup X \cup\{r\} \in \mathcal{F}$ as well, from which the claimed result then follows.

Using this representation of $K_{1}$, and letting $N=|\widetilde{X}|$, we have

$$
\left|K_{1}\right|=2^{N-k-3}
$$

and

$$
\left\|K_{1}\right\|_{\widetilde{X}}=\frac{1}{2}(N+k+3) 2^{N-k-3}
$$

From Lemma 24 we have

$$
\begin{aligned}
\|\left. K_{2}\right|_{\tilde{X}} & \geqslant \frac{1}{2}\left|K_{2}\right| \log _{2}\left(\left|K_{2}\right|\right)+k\left|K_{2}\right| \\
\|\left. K_{3}\right|_{\widetilde{X}} & \geqslant \frac{1}{2}\left|K_{3}\right| \log _{2}\left(\left|K_{3}\right|\right)+(k+1)\left|K_{3}\right| \\
\|\left. K_{4}\right|_{\widetilde{X}} & \geqslant \frac{1}{2}\left|K_{4}\right| \log _{2}\left(\left|K_{4}\right|\right)+(k+2)\left|K_{4}\right| .
\end{aligned}
$$


Combining these results, we get

$$
\begin{aligned}
\left\|[K]_{R}\right\|_{\tilde{X}}= & \left\|K_{1}||_{\tilde{X}}+\right\| K_{2}||_{\tilde{X}}+\left\|\left.K_{3}\right|_{\tilde{X}}+\right\| K_{4} \|_{\tilde{X}} \\
\geqslant & \frac{1}{2}\left((N+k+3) 2^{N-k-3}\right. \\
& +\left|K_{2}\right| \log _{2}\left(\left|K_{2}\right|\right)+2 k\left|K_{2}\right| \\
& +\left|K_{3}\right| \log _{2}\left(\left|K_{3}\right|\right)+(2 k+2)\left|K_{3}\right| \\
& \left.+\left|K_{4}\right| \log _{2}\left(\left|K_{4}\right|\right)+(2 k+4)\left|K_{4}\right|\right) .
\end{aligned}
$$

Now, in order for $\rho_{\widetilde{X}}\left([K]_{R}\right)$ to be greater than $\frac{1}{2}$, we need to show that

$$
2||[K]_{R}\left\|_{\tilde{X}}-N\left(\sum_{i=1}^{4}\left|K_{i}\right|\right)=2||[K]_{R}\right\|_{\tilde{X}}-N\left(2^{N-k-3}+\sum_{i=2}^{4}\left|K_{i}\right|\right)
$$

is greater than 0. Using (4.7), we can bound (4.8) from below by the function

$$
\begin{aligned}
(k+3) 2^{N-k-3} & +\left|K_{2}\right| \log _{2}\left(\left|K_{2}\right|\right)+(2 k-N)\left|K_{2}\right| \\
& +\left|K_{3}\right| \log _{2}\left(\left|K_{3}\right|\right)+(2 k+2-N)\left|K_{3}\right| \\
& +\left|K_{4}\right| \log _{2}\left(\left|K_{4}\right|\right)+(2 k+4-N)\left|K_{4}\right| .
\end{aligned}
$$

Since (4.9) is a convex function, we can minimize it over each of the variables $\left|K_{i}\right|$, $i=2,3,4$, independently. Thus, the global minimum is at

$$
\begin{aligned}
& \left|K_{2}\right|=2^{N-2 k-\frac{1}{\ln 2}} \\
& \left|K_{3}\right|=2^{N-2 k-2-\frac{1}{\ln 2}} \\
& \left|K_{4}\right|=2^{N-2 k-4-\frac{1}{\ln 2}} .
\end{aligned}
$$

Plugging (4.10) into (4.9) gives

$$
\begin{aligned}
& =(k+3) 2^{N-k-3}-\frac{1}{\ln 2}\left(2^{N-2 k-\frac{1}{\ln 2}}+2^{N-2 k-2-\frac{1}{\ln 2}}+2^{N-2 k-4-\frac{1}{\ln 2}}\right) \\
& =2^{N-k}\left[(k+3) 2^{-3}-\frac{2^{-k-\frac{1}{\ln 2}}}{\ln 2}\left(1+\frac{1}{4}+\frac{1}{16}\right)\right] \text {, }
\end{aligned}
$$

which is positive for any value of $k \geqslant 1$.

Note that the bounds given by Theorem 23 can be improved using the results in [3]. However, even with these improved bounds the techniques used in Lemma 26 fail when $|X| \geqslant 4$. Furthermore, if $\mathcal{F}$ is simply well-graded, but not $X$-closed, the same techniques also fail when $|X| \geqslant 3$. In any case, in its current form Lemma 26 is enough to prove our next result. 
Theorem 27. Let $X$ be a minimal set in a well-graded union-closed family $\mathcal{F}$, and assume also that $\mathcal{F}$ is $X$-closed. Then, $\mathcal{F}$ satisfies the union-closed sets conjecture if $\left|X \cup X^{\mathcal{O}}\right| \leqslant 6$.

Proof. Let $\widetilde{X}=X \cup X^{\mathcal{O}}$. From Corollary 19 we know that $\mathcal{F}$ satisfies the union-closed sets conjecture if $|\widetilde{X}| \leqslant 5$; furthermore, by Theorem 18 we know that $X^{\mathcal{O}}$ contains an abundant element if $\left|X^{\mathcal{O}}\right| \leqslant 2$. Combining this with the fact that a singleton or a doubleton always contains an abundant item, we only need to consider the case when $|X|=\left|X^{\mathcal{O}}\right|=3$.

With that in mind, consider any $K \in \mathcal{F}$. We claim that $\mathcal{F}$ satisfies the union-closed sets conjecture if $\rho_{\widetilde{X}}\left([K]_{R}\right) \geqslant \frac{1}{2}$. To see this, let $\left[K_{1}\right]_{R},\left[K_{2}\right]_{R}, \ldots,\left[K_{m}\right]_{R}$ be the set of equivalence classes associated with the equivalence relation $R$; note that these classes form a partition of $\mathcal{F}$. By assumption, for each $i=1, \ldots, m$ we have

$$
\rho_{\widetilde{X}}\left(\left[K_{i}\right]_{R}\right)=\frac{\|\left.\left[K_{i}\right]_{R}\right|_{\widetilde{X}}}{\left|\left[K_{i}\right]_{R}\right| \cdot|\widetilde{X}|} \geqslant \frac{1}{2},
$$

from which it follows that

$$
\left\|\left[K_{i}\right]_{R}\right\|_{\tilde{X}} \geqslant \frac{1}{2}\left|\left[K_{i}\right]_{R}\right| \cdot|\widetilde{X}|
$$

We then have

$$
\begin{aligned}
\|\mathcal{F}\|_{\tilde{X}} & =\sum_{i=1}^{m}\left\|\left[K_{i}\right]_{R}\right\|_{\tilde{X}} \\
& \geqslant \frac{1}{2} \sum_{i=1}^{m}\left|\left[K_{i}\right]_{R}\right| \cdot \mid \widetilde{X} \\
& =\frac{1}{2}|\mathcal{F}| \cdot|\widetilde{X}|
\end{aligned}
$$

which implies that $\rho_{\widetilde{X}}(\mathcal{F}) \geqslant \frac{1}{2}$; thus, it follows that at least one element of $\widetilde{X}$ is abundant in $\mathcal{F}$.

It remains to shows that, for any $K \in \mathcal{F}$, we have $\rho_{\widetilde{X}}\left([K]_{R}\right) \geqslant \frac{1}{2}$. By Lemma 26, the inequality holds if $K \backslash \widetilde{X} \neq \varnothing$. On the other hand, suppose $K \subseteq \tilde{X}$. Note that by (4.5), since $K \subseteq \widetilde{X}$ it follows that $L \subseteq \widetilde{X}$ for any $L \in[K]_{R}$. Now, if $|L| \leqslant 2$ then one of the elements in $L$ is abundant, and we are done. Thus, assume that $|L| \geqslant 3$ for any $L \in[K]_{R}$. We then have $|L \cap \widetilde{X}|=|L| \geqslant 3$ (i.e., every set in $[K]_{R}$ contains at least three elements of $\widetilde{X})$ which implies that $\rho_{\widetilde{X}}\left([K]_{R}\right) \geqslant \frac{1}{2}$.

\section{Acknowledgements}

The author would like to specifically acknowledge the work of Bruhn and Schaudt in [4], which served as a tremendous resource and guide in both identifying and learning many of the techniques used in this work. The author is also grateful to the anonymous reviewer for many helpful suggestions and comments. 


\section{References}

[1] A. Abdollahi, R. Woodroofe, and G. Zaimi. Frankl's conjecture for subgroup lattices. The Electronic Journal of Combinatorics, 24(3), 2017.

[2] I. Balla. Minimum density of union-closed families. arXiv:1106.0369, 2011.

[3] I. Balla, B. Bollobas, and T. Eccles. Union-closed families of sets. Journal of Combinatorial Theory (Series A), 120:531-544, 2013.

[4] H. Bruhn and O. Schaudt. The journey of the union-closed sets conjecture. Graphs and Combinatorics, 31:2043-2074, 2015.

[5] J.-P. Doignon and J.-C. Falmagne. Spaces for the assessment of knowledge. International Journal of Man-Machine Studies, 23:175-196, 1985.

[6] J.-P. Doignon and J.-C. Falmagne. Well-graded families of relations. Discrete Mathematics, 173:35-44, 1997.

[7] D. Duffus and B. Sands. An inequality for the sizes of prime filters of finite distributive lattices. Discrete Mathematics, 201:89-99, 041999.

[8] T. Eccles. A stability result for the union-closed size problem. Combinatorics, Probability and Computing, 25(3):399-418, 2016.

[9] D. Eppstein, J.-C. Falmagne, and H. Uzun. On verifying and engineering the wellgradedness of a union-closed family. Journal of Mathematical Psychology, 53(1): 34-39, 2009.

[10] J.-C. Falmagne and J.-P. Doignon. Learning Spaces. Springer-Verlag, Heidelberg, 2011.

[11] J.-C. Falmagne, E. Cosyn, J.-P. Doignon, and N. Thiéry. The assessment of knowledge, in theory and in practice. In R. Missaoui and J. Schmidt, editors, Formal Concept Analysis: Foundations and Applications, pages 61-79. Spring-Verlag, 2006.

[12] J.-C. Falmagne, D. Albert, C. Doble, D. Eppstein, and X. Hu, editors. Knowledge Spaces: Applications in Education. Springer-Verlag, Heidelberg, 2013.

[13] I. Karpas. Two results on union-closed families. arXiv:1708.01434, 2017.

[14] M. Koppen. On alternative representations for knowledge spaces. Mathematical Social Sciences, 36(2):127-143, 1998.

[15] J. Matayoshi. On the properties of well-graded union-closed families. Journal of Mathematical Psychology, 80:15-21, 2017.

[16] B. Poonen. Union-closed families. Journal of Combinatorial Theory, Series A, 59: 253-268, 1992.

[17] J. Pulaj, A. Raymond, and D. Theis. New conjectures for union-closed families. The Electronic Journal of Combinatorics, 23(3) \#P3.23, 2016.

[18] A. Raz. Note on the union-closed sets conjecture. The Electronic Journal of Combi- 
natorics, 24(3) \#P3.53, 2017.

[19] D. Reimer. An average set size theorem. Combinatorics, Probability and Computing, 12:89-93, 2003.

[20] I. Rival, editor. Graphs and order, volume 147. Springer Netherlands, 1985.

[21] D. Sarvate and J.-C. Renaud. On the union-closed sets conjecture. Ars Combinatorica, 27:149-154, 1989.

[22] P. Wójcik. Density of union-closed families. Discrete Mathematics, 105:259-267, 1992. 